\title{
The effects of sex, nation, ethnicity, age and self-reported pubertal development on participant-measured right-left 2D:4D (Dr-l) in the BBC internet study
}

\author{
J.T. Manning ${ }^{1}$, B. Fink ${ }^{2,3,4}{ }^{*}$ (D) L. Mason ${ }^{1}$, A. Kasielska-Trojan ${ }^{5}$ and R. Trivers ${ }^{6}$ \\ ${ }^{1}$ Applied Sports, Technology, Exercise, and Medicine (A-STEM), Swansea University, Swansea, United Kingdom, ${ }^{2}$ Biosocial \\ Science Information, Biedermannsdorf, Austria, ${ }^{3}$ Department of Evolutionary Anthropology, University of Vienna, Vienna, \\ Austria, ${ }^{4}$ Human Evolution and Archaeological Sciences (HEAS), University of Vienna, Vienna, Austria, ${ }^{5}$ Plastic, \\ Reconstructive and Aesthetic Surgery Clinic, Institute of Surgery, Medical University of Łódź, Łódź, Poland and \\ ${ }^{6}$ Southfield, St. Elizabeth, Jamaica \\ ${ }^{*}$ Corresponding author. Email: bfink@gwdg.de
}

(Received 3 October 2021; revised 11 December 2021; accepted 13 December 2021; first published online 28 January 2022)

\begin{abstract}
Digit ratio (2D:4D) - a proxy for prenatal sex steroids - shows sex, nationality and ethnic differences and is linked to pubertal onset. It is unclear whether right-left 2D:4D (Dr-l) also correlates with prenatal sex steroids, as evidence of these differences has been less conclusive. The present study examined the effects of sex, nation, ethnicity, age and self-reported pubertal development (i.e. the rate of physical development and age at menarche [females] or first shave [males]) on Dr-l in a large online study (the BBC internet study). Digit lengths were self-measured in 201,865 adults (110,955 males) and the sample of nations included 41 countries. Participants reported the self-perceived rate of physical pubertal development on a five-point scale (from very slow to very fast) and provided information on the age at menarche or first shave. Adult ( $>17$ years) males had lower Dr-l than females with weak effect size across 41 nations (males-females; $d=-.065, p<.0001)$. There were sex and ethnicity effects on Dr-l across seven ethnic groups with males $<$ females and lower Dr-l in Whites and Middle/Near Eastern participants compared to Asian, Black and Chinese respondents. Considering age effects, the authors focused on participants $>12$ years; there were stable sex differences and a weak positive effect of age on Dr-l. Dr-l showed a positive relationship with the rate of physical development and a negative relationship with age at menarche or first shave. Relationships were present in males and females with stronger effects in the latter. It is concluded that Dr-l shows a weak sex difference (males < females) independent of nation, ethnicity and age, and suggest that Dr-l is a proxy for prenatal sex steroids.
\end{abstract}

Keywords: testosterone and digit ratios; auxology; body image

\section{Introduction}

Digit ratio (2D:4D) - the relative lengths of the second (2D) and fourth (4D) fingers - is thought to be a negative correlate of prenatal testosterone $(\mathrm{T})$ and a positive correlate of prenatal oestrogen (E) (Zheng \& Cohn, 2011; Manning, 2011). The first detailed consideration of these associations reported: i) 2D:4D was sexually dimorphic (males $<$ females), ii) it showed stability across the age range of 2 to 25 years, and iii) its associations with target traits (in this case aspects of spermatogenesis) were strongest for right $2 \mathrm{D}: 4 \mathrm{D}$. The latter observation led to the suggestion that right 
2D:4D was more sensitive to prenatal sex steroids than left 2D:4D (Manning et al., 1998). Many later studies have found right 2D:4D to be lower than left 2D:4D and more sexually dimorphic (for meta-analysis see Hönekopp \& Watson, 2010). The side difference in 2D:4D suggests that the magnitude of right-left 2D:4D (Dr-l) may also be a correlate of prenatal sex steroids. This possibility was first suggested in connection with hand preference (Manning et al., 2000) and considered in detail by Manning (2002; for overall sex differences p. 21-22, spermatogenesis p. 27-30, waist-to-hip ratio p. 32-37, oestrogen levels p. 37-38). Subsequently, low Dr-l (putatively high prenatal T and low prenatal E) has been linked to a diverse set of sex-dependent traits such as the implicit need for power (Schultheiss et al., 2019) and elite performance in rugby (Bennett et al., 2010).

Side differences in sexually dimorphic traits have been noted by Tanner (1990), who suggested that in humans such traits tend to be more intensely expressed in the "male form" on the right side of the body and in the "female form" on the left. Such lateralization can be seen in true hermaphrodites in which the ovary is more often found on the left side of the body (Mittwoch \& Mahadevaiah, 1980). It also extends to the directional asymmetry of cognitive abilities. Men and women with right-larger gonads return high scores in male-favouring cognitive tasks and high scores in female-favouring tasks are linked to left-larger gonads in both sexes (Kimura, 1994). Patterns of development may shed light on the early origin of such side differences in ontogeny. In a mouse model, a molecular marker of cartilage differentiation (Sox9) shows sex differences in right paw 2D:4D (males < females) by embryonic day 17 but 2D:4D dimorphism is not found in the left paw. However, by embryonic day 21, both right and left paws show significant 2D:4D sex-dimorphism (Zheng \& Cohn, 2011). This indicates that the sexually dimorphic $2 \mathrm{D}: 4 \mathrm{D}$ ratio in mice develops during a narrow window in early ontogeny and that the developmental rate of dimorphism differs between the right and left sides. Of relevance to the prenatal determination of Dr-1, Baxter et al. (2020) have reported associations between maternal sex steroids and offspring 2D:4D in a New World primate (Titi monkeys). In this species, sex differences in right 2D:4D are similar to that of humans (males $<$ females). Maternal urine samples were collected 15-20 weeks before birth and assayed for T and E. High maternal concentrations of $\mathrm{T}$ and low levels of $\mathrm{E}$ were associated with low Dr-l.

In common with 2D:4D, the Dr-l may also be sexually dimorphic in humans (males $<$ females) (e.g. Manning, 2002, p. 27-30; Manning \& Fink, 2008). However, the evidence for the sex difference in Dr-1 is mixed with some samples showing no dimorphism (e.g. Manning, 2002, p. 21-22; Putz et al., 2004), and little is known about its variation among nations and ethnic groups, whether it is stable with age and whether it relates to patterns of development. The present study considers Dr-l in the context of these variables using data from a large online sample (the British Broadcasting Company [BBC] internet study).

Previous research has documented associations between 2D:4D, Dr-l and prenatal sex steroids (see Breedlove, 2010 for a review), including more recent research on associations between Dr-1 and single-nucleotide polymorphisms (Zhanbing et al., 2019; Zhang et al., 2021). However, these studies shed little light on whether Dr-l is sexually dimorphic and whether it correlates with many sexually dimorphic behavioural and physiological traits. Manning et al. (2007) and Manning and Fink (2008) have considered sex differences in a large $(n>255,000)$ sample of self-reported 2D:4D and Dr-l in the BBC internet study. Both 2D:4D and Dr-l were sexually dimorphic (males $<$ females) with an effect size for the former of right hand $d=.20$, left hand $d=.15$ and the latter $d=.07$, all $p<.0001$. These effect sizes are attenuated by imprecise finger measurement due to self-reported surveys. For example, mean sex differences in experimenter-measured 2D:4D are approximately $d=.40$ to .60 (Manning, 2002). However, it is important to note that effect sizes for sex differences in Dr-l are less than half for those of 2D:4D. Perhaps, as a consequence of such small effect sizes, it remains unclear whether sexual dimorphism in Dr-l is widespread within and between national and ethnic groups.

Concerning correlations between Dr-l and sexually dimorphic traits, there are several examples in the literature for sex-dependent diseases and their links with patterns of development. One such 
is the association between breast cancer, left-handedness and Dr-l. Muller et al. (2012) reported evidence from patterns of 2D:4D and Dr-l that suggested high prenatal $\mathrm{T}$ and $\mathrm{E}$ were linked to an increase in breast cancer risk. Left-handedness is associated with high prenatal $\mathrm{T}$ and increased risk of breast cancer in women (Ramadhani et al., 2005) and low Dr-l (high prenatal T) is related to a left-hand preference (Manning et al., 2000; Manning \& Peters, 2009). Early age at menarche has also been linked to increased risk of breast cancer and 2D:4D, particularly right-hand 2D:4D, has been reported to be negatively associated with age at menarche (e.g. Matchock, 2008; Manning \& Fink, 2011). In this regard, Dr-l may be associated with developmental patterns during puberty, including age at initiation of puberty in both girls and boys.

The present study explores the effects of sex, nation, ethnicity, age and self-perceived rate of physical development during puberty on participant-measured Dr-l. As a working hypothesis, it is assumed that Dr-l is a negative correlate of prenatal $\mathrm{T}$ and a positive correlate of prenatal $\mathrm{E}$. Thus, Dr-l should show a sex difference (males $<$ females) that is widespread across nations and ethnic groups and stable across age groups. Concerning the measures of pubertal development (selfperceived rate of physical development and age at menarche [females] or age that shaving began [males]), there is evidence that the timing of puberty is one correlate of prenatal androgenization. For example, high prenatal $\mathrm{T}$ is linked to late spermarche in boys (Cohen-Bendehan et al., 2005) and animal models have confirmed this association in males (Dela Cruz \& Pereira, 2012). Therefore, it is predicted that high Dr-l will be linked to faster rates of self-perceived physical pubertal development across nations and ethnic groups.

\section{Methods}

The BBC internet study was a multi-ethnic and multi-national survey, hosted by the BBC on their Science and Nature website. It comprised around 200 questions concerning cognitive and behavioural tests and included information on demographics, personality, sexual behaviour, and physical characteristics, such as 2D:4D (see Reimers, 2007 for further details). A total sample of 255,116 participants completed all study tasks. In addition to ethnicity (Asian/Asian British, Black/Black British, Black other, Chinese, Middle/Near Eastern, Mixed Ethnic, White), participants provided information about their sex (male or female), age (integer 0 to 99 years), and where they resided (the United Kingdom, then 240 other countries). The predominant ethnicity was "White" (reported by $84.1 \%$ of participants), and the most commonly represented nations were the United Kingdom (46.9\%), the United States (27.7\%), Canada (5.2\%), and Australia (3.6\%) with 11 other nations represented by $n>1,000$ participants.

Participants responded to a two-question item concerning their self-perceived physical developmental rate at puberty. The questions were phrased as follows:

"Compared to your classmates at school, was your rate of physical development, e.g. facial hair, breast development, during puberty". Response options ranged from 1 (very slow) to 5 (very fast).

"How old were you when you started puberty (either when you started your first period or when you started shaving?)" Response options ranged up to 999 years old and these included some extreme values ( 0 years to 132 years). Therefore, the authors followed Manning and Fink (2011) in restricting the analyses to a range of 8 years to 17 years.

Participants self-measured 2D and 4D of right and left hands using the methodology of Manning et al. (1998). They viewed a diagram of the hand and were instructed to measure their fingers on the ventral side of the digit from the fingertip to the most proximal crease with a conventional ruler. Measurements were reported to the nearest millimetre using dropdown menus. The 2D:4D was calculated by dividing the $2 \mathrm{D}$ by $4 \mathrm{D}$ digit lengths and $\mathrm{Dr}-1$ (the difference of rightminus left-hand 2D:4D) resulted from subtracting left from right 2D:4D. In the present study, the initial analyses were restricted to adults ( $>17$ years) and in further analyses, considering the effect of age on Dr-l, the participants were $>12$ years. As in earlier reports, the tails of the 2D:4D distributions were removed by considering right and left $2 \mathrm{D}: 4 \mathrm{D}$ within the range of $\geq 0.80$ to $\leq 1.20$. 


\section{Results}

\section{Descriptive statistics}

The adult sample comprised 201,865 participants (110,955 males) of ages 18 to 99 years $(M=31.78, S D=11.08)$ and the national sample included participants from 41 countries.

Dr-l in the adult sample ranged from -.400 to .400 with a mean of $.001(S D=.042)$. There was no indication for right or left skew of the Dr-l distribution $($ skew $=.193)$ but it appeared leptokurtic with a higher and sharper central peak compared to the normal distribution (kurtosis = 7.921).

\section{Sex and ethnic differences}

There was a sex differences for Dr-l in the total sample and across nations with males having smaller values than females (males $M=-.001, S D=.040$; females $M=.002, S D=.043$, $F[1,201863]=195.61, p<.0001)$. Table 1 reports descriptive statistics of male and female Dr-1 by nation together with effect sizes (Cohen's $d$ ) for the sex difference (Figure 1). The mean effect size (male Dr-1 - female Dr-1) was $d=-.065$ (a negative value denotes Dr-l male $<$ female) and this differed from zero $(t[40]=4.88, p<.0001)$.

With regard to ethnicity in the total sample, mean (SD's and SE's) values of Dr-1 for seven ethnic groups are given in Table 2. Effect sizes for the sex differences varied from $d=-.05$ (Chinese) to -.12 and -.10 (Black/Black British and Black other, respectively). All values of $d$ were negative, denoting lower mean Dr-l in males compared to females. A 2 (sex) 7 (ethnicity) ANOVA with Dr-l as dependent variable showed an effect of $\operatorname{sex}(F[1,200597]=32.45, p<.0001)$ and ethnicity $(F[6,200597]=8.84, p<.0001)$ but no interaction of sex ${ }^{\star}$ ethnicity $(F[6,200597]=$ $0.83, p=.549)$. The mean sex difference was .003 independent from ethnicity. Whites and Middle/ Near Eastern participants had the lowest mean Dr-l and Black participants had the highest mean Dr-l values. Significant differences were found for comparisons between Asian/Asian British vs. Black/Black British (mean difference $=-.002, p<.05)$, Black other $(-.003, p<.01)$, and White $(.001, p<.01)$ participants; for Black/Black British vs. Middle/Near Eastern $(.004, p<.01)$ and White $(.004, p<.01)$ participants; for Black other vs. Middle/Near Eastern (.005, $p<.001)$, Mixed Ethnic $(.003, p<.05)$, and White $(.004, p<.0001)$ participants; for Chinese vs. Middle/Near Eastern $(.003, p<.01)$, and White $(.003, p<.0001)$ participants; for Middle/Near Eastern vs. Mixed Ethnic $(-.002, p<.05)$ participants; and for Mixed Ethnic vs. White $(.001, p<.01)$ participants. The absence of an interaction between sex and ethnicity suggests that the effects on Dr-l were independent from one another.

\section{Sex and age effects}

To examine the effects of sex and age on Dr-l, the authors considered participants $>12$ years of age. The analysis focused on year groups with $n>1,000$ participants to maintain sufficient statistical power; thus, in the data set this included participants 13 to 57 years (Table 3).

A 2 (sex) x 45 (age) ANOVA with Dr-l as dependent variable showed an effect of sex $(F[1,227052]=121.80, p<.0001)$ and a much smaller (but significant) effect of age $(F[44,227052]=1.88, p<.001)$ but no interaction of sex $*$ age $(F[44,227052]=0.87$, $p=.711$ ). Males had lower Dr-l than females (mean difference $=.003$ ). The absence of an interaction between sex and age suggests that the effects on Dr-l were independent from one another.

Because the prior analysis of ethnicity influences on Dr-l showed a small but significant effect, the authors performed the analysis of sex and age effects on Dr-l again, this time restricting the sample to the largest ethnic group, i.e. White participants. There was an effect of sex $(F[1,191385]=110.80, p<.0001)$ and age $(F[44,191385]=1.88, p<.001)$ but no interaction of sex ${ }^{*}$ ethnicity $(F[44,191385]=0.93, p=.608)$. Thus, the effects of sex and age are essentially the same in the total sample and for White participants only. 
Table 1. Descriptive statistics (sample sizes, means and SD's) for male and female Dr-l in 41 nations. A negative value of Cohen's $d$ effect size denotes the male mean Dr-I is lower than the female mean Dr-l

\begin{tabular}{|c|c|c|c|c|c|c|c|}
\hline \multirow[b]{2}{*}{ Nations } & \multicolumn{3}{|c|}{ Males } & \multicolumn{3}{|c|}{ Females } & \multirow[b]{2}{*}{$d$} \\
\hline & $n$ & $M$ & $S D$ & $n$ & $M$ & $S D$ & \\
\hline Argentina & 125 & .002 & .045 & 78 & -.002 & .048 & .090 \\
\hline Australia & 4103 & -.001 & .039 & 3690 & .002 & .039 & -.080 \\
\hline Austria & 213 & -.005 & .042 & 170 & -.004 & .037 & -.030 \\
\hline Belgium & 764 & -.003 & .036 & 499 & $6.000 \mathrm{E}-5$ & .041 & -.080 \\
\hline Brazil & 170 & -.001 & .046 & 109 & .003 & .040 & -.090 \\
\hline Bulgaria & 172 & .001 & .043 & 186 & -.003 & .037 & .100 \\
\hline Canada & 5723 & .004 & .038 & 5279 & .002 & .043 & -.040 \\
\hline China & 169 & $2.000 \mathrm{E}-5$ & .036 & 124 & .004 & .044 & -.100 \\
\hline Croatia & 95 & -.003 & .030 & 102 & .001 & .027 & -.140 \\
\hline Czech & 146 & -.002 & .030 & 94 & $3.000 \mathrm{E}-4$ & .033 & -.070 \\
\hline Denmark & 380 & -.006 & .034 & 347 & -.003 & .036 & -.090 \\
\hline Finland & 875 & -.001 & .035 & 675 & $2.000 \mathrm{E}-4$ & .035 & -.030 \\
\hline France & 535 & -.004 & .035 & 376 & .004 & .034 & -.230 \\
\hline Germany & 866 & -.002 & .038 & 546 & .002 & .034 & -.110 \\
\hline Greece & 420 & -.001 & .037 & 392 & -.001 & .039 & .000 \\
\hline Hungary & 97 & -.003 & .027 & 103 & .004 & .035 & -.220 \\
\hline Iceland & 85 & -.004 & .025 & 88 & -.001 & .052 & -.070 \\
\hline India & 2274 & $5.000 \mathrm{E}-5$ & .040 & 577 & .005 & .047 & -.120 \\
\hline Ireland & 2307 & -.001 & .039 & 2177 & $-4.000 E-4$ & .044 & -.010 \\
\hline Israel & 195 & $4.000 \mathrm{E}-4$ & .034 & 131 & .004 & .038 & -.121 \\
\hline Italy & 248 & -.002 & .032 & 166 & .005 & .041 & -.190 \\
\hline Japan & 289 & .002 & .038 & 163 & .004 & .039 & -.050 \\
\hline Malaysia & 418 & $4.000 \mathrm{E}-4$ & .034 & 350 & .001 & .043 & -.040 \\
\hline Mexico & 208 & $-2.000 \mathrm{E}-4$ & .041 & 131 & .005 & .045 & -.120 \\
\hline N Zealand & 970 & -.002 & .038 & 954 & .003 & .040 & -.130 \\
\hline Netherlands & 1172 & -.004 & .039 & 798 & -.002 & .039 & -.050 \\
\hline Norway & 305 & -.003 & .032 & 213 & .001 & .040 & -.110 \\
\hline Pakistan & 245 & -.001 & .041 & 72 & -.002 & .040 & .020 \\
\hline Phillipines & 190 & .003 & .045 & 201 & .001 & .052 & .040 \\
\hline Poland & 197 & -.005 & .042 & 225 & .002 & .044 & -.160 \\
\hline Portugal & 187 & .001 & .042 & 139 & .002 & .039 & -.040 \\
\hline Romania & 166 & .001 & .042 & 173 & -.004 & .035 & .130 \\
\hline Russia & 88 & -.010 & .036 & 96 & -.006 & .043 & -.100 \\
\hline Singapore & 817 & .002 & .040 & 944 & .003 & .041 & .120 \\
\hline Spain & 468 & -.001 & .045 & 289 & .003 & .039 & -.090 \\
\hline
\end{tabular}


Table 1. (Continued)

\begin{tabular}{|c|c|c|c|c|c|c|c|}
\hline \multirow[b]{2}{*}{ Nations } & \multicolumn{3}{|c|}{ Males } & \multicolumn{3}{|c|}{ Females } & \multirow[b]{2}{*}{$d$} \\
\hline & $n$ & $M$ & $S D$ & $n$ & $M$ & $S D$ & \\
\hline Sweden & 760 & .001 & .037 & 386 & .002 & .038 & -.030 \\
\hline Switzerland & 323 & .001 & .034 & 206 & .004 & .040 & -.080 \\
\hline Turkey & 653 & $9.000 \mathrm{E}-5$ & .039 & 595 & $-2.000 \mathrm{E}-4$ & .041 & .007 \\
\hline UK & 51324 & -.001 & .040 & 40207 & .002 & .042 & -.070 \\
\hline UAE & 169 & -.002 & .032 & 117 & .008 & .050 & -.240 \\
\hline USA & 24571 & $2.000 \mathrm{E}-4$ & .043 & 21198 & .003 & .046 & -.060 \\
\hline
\end{tabular}

\section{Pubertal physical development}

The self-perceived rate of pubertal physical development is a subjective measure and that of age at menarche/first shave is an objective measure. To establish whether the former is related to the latter, the authors examined relationships between the two variables. For females $(n=88,963)$, the correlation was $r=-.58, p<.0001$. For males $(n=102,572)$, it was $r=-.38, p<.0001$.

A series of linear regression analyses were performed with self-perceived pubertal physical development (females and males), age at menarche (females) or first shave (males) as the dependent variables, and Dr-1, right or left 2D:4D together with participant's age, as independent variables. These analyses were conducted separately for female and male participants, first with the total sample and then for White participants only.

In females $(n=90,031)$, Dr-1 $(B=.24, S E B=.08$, beta $=.01, p<.01)$ and participant's age $(B=-.004, S E B=.0003$, beta $=-.04, p<.0001)$ predicted self-perceived pubertal physical developmental rate albeit in opposite directions (i.e. a positive association for the former and a negative association for the latter). Dr-l was negatively associated with age at menarche $(n=89,228 ; B=-.34, S E B=.11$, beta $=-.01, p<.01)$ and positively with participant's age $(B=.005, S E B=.0005$, beta $=.04, p<.0001)$. Similar findings were obtained with right $2 \mathrm{D}: 4 \mathrm{D}$ for self-perceived pubertal physical developmental rate $(B=.44, S E B=.07$, beta $=.02$, $p<.0001$; participant's age $B=-.004, S E B=.0003$, beta $=-.04, p<.0001)$ and age at menarche $(B=-.37, S E B=.02$, beta $=-.01, p=.0001$; participant's age $B=.005, S E B=.0005$, beta $=.04$, $p<.0001)$. Left 2D:4D was also positively related to self-perceived pubertal physical developmental rate $(B=.29, S E B=.07$, beta $=.01, p<.0001$; participant's age $B=-.004, S E B=.0003$, beta $=-.04, p<.0001)$ but not to age at menarche $(B=-.14, S E B=.10$, beta $=-.005, p=.16$; participant's age $B=.005, S E B=.0005$, beta $=.04, p<.0001)$.

In common with females, male Dr-l was positively related to self-perceived pubertal physical developmental rate $(n=109,723 ; B=.15, S E B=.07$, beta $=.01, p<.05$; participant's age $B=-.003$, SE $B=.0003$, beta $=-.04, p<.0001)$ and negatively related to age a first shave $(n$ $=102,785, B=-.38, S E B=.12$, beta $=-.010, p<.01$; participant's age $B=.02$, SE $B=.0004$, beta $=.11, p<.0001)$. Similarly, right $2 \mathrm{D}: 4 \mathrm{D}$ was positively associated with selfperceived pubertal physical developmental rate $(B=.14, S E B=.06$, beta $=.01, p<.05$; age $B=-.003, S E B=.0003$, beta $=-.04, p<.0001)$ and negatively related to age at first shave $(B=-.22$, SE $B=.099$, beta $=-.01, p<.05)$. In contrast, left $2 \mathrm{D}: 4 \mathrm{D}$ was not related to self-perceived pubertal physical developmental rate $(B=.05, S E B=.06$, beta $=.001, p=.431$; participant's age $B=-.003, S E B=.0003$, beta $=-.04, p<.0001)$, nor was it correlated with age at first shave $(B=.04, S E B=.10$, beta $=.001, p=.70$; participant's age $B=.02, S E B=.0004$, beta $=.11$, $p<.0001)$.

Considering White participants only revealed essentially the same findings as with the total sample. The analyses were as follows: 


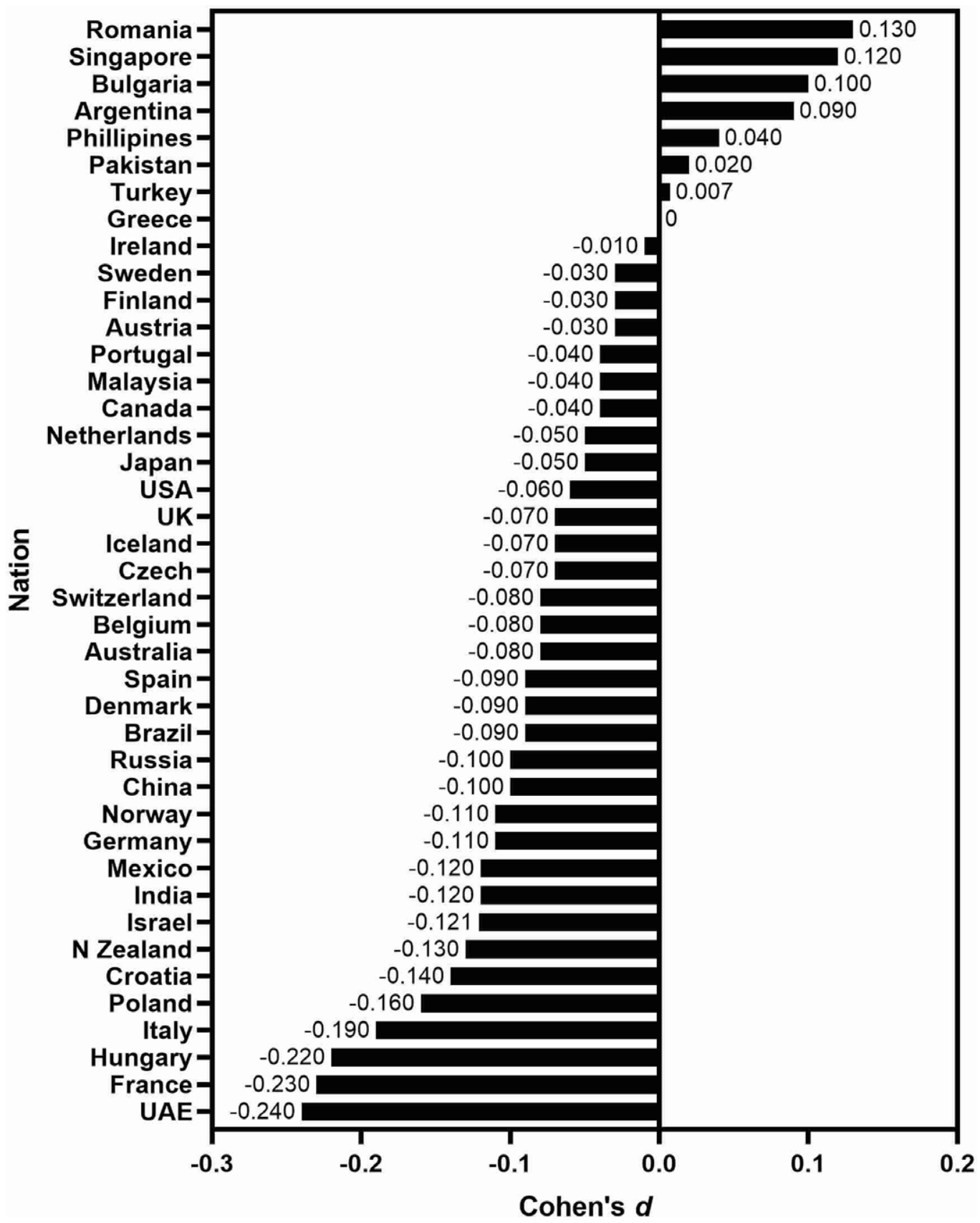

Figure 1. Effect sizes (Cohen's $d$ ) for the sex difference in right-left 2D:4D (Dr-l) from participants of 41 nations.

For females $(n=76,835)$, Dr-l $(B=.25, S E B=.09$, beta $=.01, p<.01)$ and participant's age $(B=-.004, S E B=.0003$, beta $=-.05, p<.0001)$, were significantly related to self-perceived pubertal physical development rate and age. Dr-l was negatively associated with age at menarche $(n=76,188 ; B=-.38, S E B=.12$, beta $=-.010 ; p<.01)$ and positively with participant's age $(B=.003, S E B=.0005$, beta $=.03, p<.0001)$. Similar findings were again obtained with right 2D:4D (pubertal physical developmental rate, $B=.42$, SE $B=.07$, beta $=.02, p<.0001$; participant's 
Table 2. Descriptive statistics (sample sizes, means, SD's and SE's) of Dr-l split by sex in seven ethnic groups. Values of $t$ and $p$ for sex differences and effect sizes (Cohen's $d$ ) are given for each ethnicity. Negative values of $d$ denote lower mean Dr-l in males compared to females

\begin{tabular}{|c|c|c|c|c|c|c|c|c|c|c|c|}
\hline \multirow[b]{2}{*}{ Ethnicity } & \multicolumn{4}{|c|}{ Males } & \multicolumn{4}{|c|}{ Females } & \multirow[b]{2}{*}{$t$} & \multirow[b]{2}{*}{$p$} & \multirow[b]{2}{*}{$d$} \\
\hline & $n$ & $M$ & $S D$ & $S E$ & $n$ & $M$ & $S D$ & SE & & & \\
\hline Asian & 7958 & .0003 & .041 & .0005 & 4617 & .004 & .042 & .001 & 4.36 & $<.0001$ & -.09 \\
\hline $\begin{array}{l}\text { Black/ } \\
\text { Black British }\end{array}$ & 612 & .001 & .043 & .002 & 651 & .007 & .053 & .002 & 1.98 & .048 & -.12 \\
\hline Black other & 758 & .002 & .047 & .002 & 662 & .007 & .052 & .002 & 1.93 & .054 & -.10 \\
\hline Chinese & 2148 & .002 & .040 & .001 & 2209 & .004 & .042 & .001 & .983 & .33 & -.05 \\
\hline $\begin{array}{l}\text { Middle/ } \\
\text { Near Eastern }\end{array}$ & 1463 & -.001 & .038 & .001 & 882 & .002 & .044 & .001 & 1.73 & .08 & -.07 \\
\hline White & 93836 & -.001 & .040 & .0001 & 77462 & .002 & .043 & .0002 & 12.66 & $<.0001$ & -.07 \\
\hline Mixed Ethnic & 3603 & .0004 & .043 & .001 & 3750 & .003 & .046 & .001 & 2.59 & .0095 & -.06 \\
\hline
\end{tabular}

age $B=-.004, S E B=.0003$, beta $=-.04, p<.0001)$ and age at menarche $(B=-.47, S E B=.10$, beta $=-.02, p .<0001$; participant's age, $B=.003, S E B=.0005$, beta $=.03, p<.0001$ ), and with left $2 \mathrm{D}: 4 \mathrm{D}$ (pubertal physical development rate, $B=.27$, SE $B=.08$, beta $=.01, p<.001$; participant's age $B=-.004, S E B=.0003$, beta $=-.05, p<.0001)$, and age at menarche $(B=-.22, S E B=.11$, beta $=$ $-.008, p<.05$; participant's age $(B=.003, S E B=.0005$, beta $=.03, p<.0001)$.

For males $(n=93,003)$, Dr-1 $(B=.22$, $S E B=.08$, beta $=.01, p<.01)$ and participant's age $(B=-.003, S E B=.0003$, beta $=-.04, p<.0001)$ predicted self-perceived pubertal physical development in the same directions as in females. Age at first shave was negatively related to Dr-l $(n=88,024, B=-.39, S E B=.13$, beta $=-.01, p<.05)$ and positively associated with participant's age $(B=.17, S E B=.0005$, beta $=13, p<.0001)$. Similarly, right $2 \mathrm{D}: 4 \mathrm{D}$ was positively associated with self-perceived pubertal development $(B=.14, S E B=.07$, beta $=.01, p<.05$; participant's age $B=-.003, S E B=.0003$, beta $=-.04, p<.0001)$ and negatively with age at first shave $(B=-.30, S E B=.11$, beta $=-.009, p<.01$; participant's age $B=.17$, $S E B=.0005$, beta $=.13, p=<.0001)$. There were no significant relationships between left 2D:4D and self-perceived pubertal development $(B=-.001$, SE $B=.07$, beta $=$ $.00007, p=.982$; participant's age $B=-.003$, $S E B=.0003$, beta $=-.04$, $p<.0001)$, or age at first shave $(B=-.03, S E B=.11$, beta $=.001, p=.76$; participant's age $B=.02$, $S E B=.0005$, beta $=.13, p<.0001)$.

Therefore, high values of Dr-l were associated with faster rates of self-perceived physical development (females and males; Figure 2), earlier menarche (females) or earlier age at first shave (males) and this applied to participants in the total sample and for White participants only.

\section{Discussion}

The present study found that Dr-l from participant-measured digit lengths is sexually dimorphic such that, in comparison to females, males tend to have lower 2D:4D in their right hand compared to their left (male Dr-1 < female Dr-1). The dimorphism remained significant after the effect of age was removed. The effect size of the sex difference (males-females) across 41 nations was $d=-.065$, i.e. it is less than that for $2 \mathrm{D}: 4 \mathrm{D}$ (right hand $d=2.0$, left hand $d=.15$ ). Across the national sample, there was one country with no difference in male-female Dr-l (Greece, $d=0)$, seven nations with male $>$ female Dr-l (ranging from $d=-.007$ [Turkey] to .130 [Romania], and 33 nations with male $<$ female Dr-l (ranging from $d=-.010$ [Ireland] to -.240 [United Arab Emirates]). Further analysis revealed an effect of ethnicity with low Dr-l in Whites and Middle- 
Table 3. Descriptive statistics (sample sizes, means, SD's and SE's) of Dr-l and sample sizes split by sex in 45 year groups (ages 13 years to 57 years)

\begin{tabular}{|c|c|c|c|c|c|c|c|c|}
\hline \multirow[b]{2}{*}{ Age (years) } & \multicolumn{4}{|c|}{ Males } & \multicolumn{4}{|c|}{ Females } \\
\hline & $n$ & $M$ & $S D$ & $S E$ & $n$ & $M$ & $S D$ & $S E$ \\
\hline 13 & 882 & .003 & .057 & .002 & 1686 & .004 & .061 & .001 \\
\hline 14 & 1583 & .003 & .056 & .001 & 2783 & .004 & .059 & .001 \\
\hline 15 & 2551 & .001 & .052 & .001 & 4152 & .002 & .056 & .001 \\
\hline 16 & 3673 & .0002 & .049 & .001 & 5638 & .004 & .052 & .001 \\
\hline 17 & 3483 & .001 & .045 & .001 & 4342 & .004 & .050 & .001 \\
\hline 18 & 4137 & -.0003 & .045 & .001 & 4589 & .002 & .047 & .001 \\
\hline 19 & 4097 & .0004 & .044 & .001 & 4123 & .003 & .046 & .001 \\
\hline 20 & 4541 & .0002 & .043 & .001 & 4487 & .002 & .044 & .001 \\
\hline 21 & 5010 & -.0004 & .040 & .001 & 4527 & .004 & .046 & .001 \\
\hline 22 & 4903 & -.001 & .039 & .001 & 4254 & .002 & .045 & .001 \\
\hline 23 & 4940 & -.0001 & .040 & .001 & 4478 & .002 & .043 & .001 \\
\hline 24 & 4930 & -.0002 & .038 & .001 & 4398 & .003 & .042 & .001 \\
\hline 25 & 5224 & -.001 & .041 & .001 & 4462 & .003 & .043 & .001 \\
\hline 26 & 4586 & -.001 & .039 & .001 & 4130 & .002 & .042 & .001 \\
\hline 27 & 4339 & .0001 & .039 & .001 & 3573 & .002 & .043 & .001 \\
\hline 28 & 4130 & .0003 & .038 & .001 & 3488 & .002 & .042 & .001 \\
\hline 29 & 3848 & .00001 & .038 & .001 & 3189 & .002 & .039 & .001 \\
\hline 30 & 3926 & -.001 & .039 & .001 & 3138 & .002 & .041 & .001 \\
\hline 31 & 3248 & -.001 & .039 & .001 & 2551 & .001 & .038 & .001 \\
\hline 32 & 3491 & -.0002 & .038 & .001 & 2713 & .001 & .040 & .001 \\
\hline 33 & 3201 & -.001 & .040 & .001 & 2370 & .003 & .042 & .001 \\
\hline 34 & 3169 & .001 & .037 & .001 & 2354 & .001 & .043 & .001 \\
\hline 35 & 3220 & .0003 & .039 & .001 & 2200 & .002 & .042 & .001 \\
\hline 36 & 2591 & -.001 & .039 & .001 & 1961 & .002 & .041 & .001 \\
\hline 37 & 2403 & -.001 & .037 & .001 & 1843 & .001 & .038 & .001 \\
\hline 38 & 2359 & -.002 & .039 & .001 & 1665 & .0003 & .043 & .001 \\
\hline 39 & 2109 & -.002 & .037 & .001 & 1526 & .002 & .042 & .001 \\
\hline 40 & 2369 & -.0002 & .039 & .001 & 1725 & .002 & .044 & .001 \\
\hline 41 & 1781 & -.002 & .036 & .001 & 1390 & .002 & .041 & .001 \\
\hline 42 & 1885 & -.0002 & .042 & .001 & 1315 & .003 & .043 & .001 \\
\hline 43 & 1606 & -.002 & .038 & .001 & 1229 & .002 & .044 & .001 \\
\hline 44 & 1649 & -.001 & .040 & .001 & 1196 & .003 & .045 & .001 \\
\hline 45 & 1591 & -.0002 & .043 & .001 & 1165 & .001 & .044 & .001 \\
\hline 46 & 1287 & -.005 & .040 & .001 & 1040 & .001 & .039 & .001 \\
\hline 47 & 1413 & -.0004 & .039 & .001 & 1061 & .002 & .041 & .001 \\
\hline
\end{tabular}


Table 3. (Continued)

\begin{tabular}{|c|c|c|c|c|c|c|c|c|}
\hline \multirow[b]{2}{*}{ Age (years) } & \multicolumn{4}{|c|}{ Males } & \multicolumn{4}{|c|}{ Females } \\
\hline & $n$ & $M$ & $S D$ & SE & $n$ & $M$ & $S D$ & $S E$ \\
\hline 48 & 1192 & -.001 & .042 & .001 & 942 & .002 & .042 & .001 \\
\hline 49 & 1145 & -.002 & .042 & .001 & 827 & .002 & .051 & .002 \\
\hline 50 & 1362 & -.001 & .042 & .001 & 1019 & .002 & .047 & .001 \\
\hline 51 & 917 & .002 & .042 & .001 & 712 & .001 & .046 & .002 \\
\hline 52 & 1038 & -.001 & .042 & .001 & 732 & .004 & .041 & .002 \\
\hline 53 & 821 & .001 & .042 & .001 & 600 & .002 & .044 & .002 \\
\hline 54 & 761 & .002 & .046 & .002 & 601 & .002 & .043 & .002 \\
\hline 55 & 819 & -.001 & .048 & .002 & 524 & .002 & .046 & .002 \\
\hline 56 & 696 & .001 & .047 & .002 & 450 & .005 & .044 & .002 \\
\hline 57 & 656 & -.0005 & .044 & .002 & 432 & .0001 & .041 & .002 \\
\hline
\end{tabular}

Figure 2. The relationship between rightleft 2D:4D (Dr-l) and self-perceived physical development in males and females.

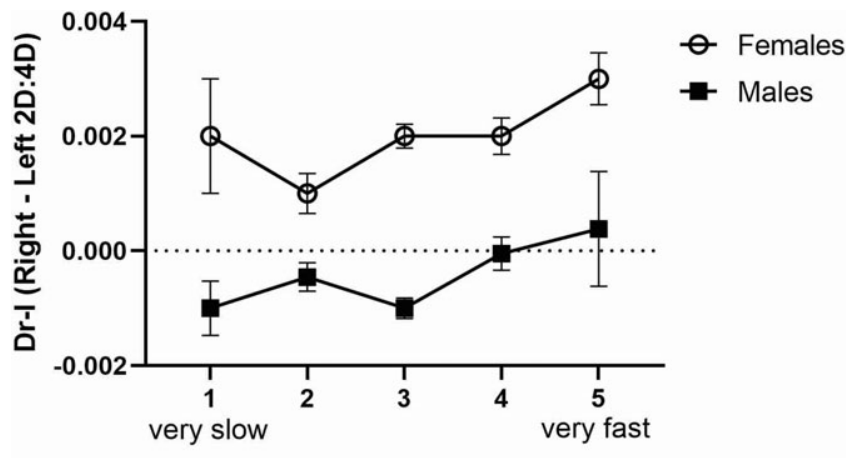

Self-Perceived Pubertal Physical Developmental Rate

Eastern peoples, intermediate values among Asian and Chinese groups, and high Dr-l in Black participants. The self-perceived puberty rate was negatively correlated with the reported age at menarche in females $(r=-.58)$ and the age at first shave in males $(r=-.38)$. Menarche is a developmental milestone that may be more memorable than the first shave, and this may account for the sex difference in the strength of these associations. The self-perceived pubertal physical developmental rate was associated with Dr-l and onset of puberty (age at menarche/first shave) in both males and females. It is to be noted that the former measure of puberty timing is a relative one that may be more appropriate for comparisons within groups such as nations. It is less relevant to comparisons within ethnic groups. Age at menarche/first shave is an absolute variable that can be used across nations and ethnic groups. Therefore, the stability of the sex difference in Dr-l in both nations and ethnic groups is relevant to self-perceived and absolute rates of pubertal development.

A pattern of sexual dimorphism that is widespread across nations, independent of ethnic groups and age, and which correlates with the rate of physical development suggests Dr-l is a universal human marker for sex steroids. The evidence indicates that low Dr-l is associated with high $\mathrm{T}$ and low E, while high Dr-1 correlates with low $\mathrm{T}$ and high E. In this regard, Dr-1 shares many of its properties with 2D:4D. Whether it is also a correlate of prenatal sex steroids which 
retains its information across all age groups remains somewhat of an open question because the data of the present study did not extend to participants younger than 13 years. However, low Dr-1 is linked to left-lateralized hand preference in Afro-Caribbean children (Manning et al., 2000) in addition to a left-hand preference for writing across nations and ethnicities in the BBC internet study (Manning \& Peters, 2009). This suggests that Dr-l is an early correlate of sex-dependent behaviours that include handedness.

In addition to containing information about prenatal sex steroids, the Dr-1 may also correlate with androgen production in challenging situations (Manning et al., 2014). Thus, the organizational effects of early exposure to androgen and oestrogen may prime the endocrine system to respond to aggressive challenges. Such challenges may provoke short-term spikes of $\mathrm{T}$ which are themselves associated with increases in strength (Kilduff et al., 2013; Crewther et al., 2015; Crewther \& Cook, 2019). These situations are regularly met with during sporting encounters. Performance in aggressive contact sports (e.g. rugby) has been reported to be linked to Dr-l. Players with low Dr-l are often chosen from their squad to represent their first team and their nation and are prolific try scores (Bennett et al., 2010). Competitive situations, such as track races, also represent a challenge for which endurance is important. Low Dr-1 has been reported to correlate with high maximal oxygen uptake (VO2max), high velocity at VO2max, and high maximum lactate concentration (Hill et al., 2012). It is not known whether the association between Dr-l and such endurance measures is correlated with T changes in response to competitive challenges. A note of caution must be sounded here, in that links between Dr-l and androgen responses to challenge have not always been replicated (e.g. Ribeiro et al., 2016). Non-replication of links between Dr-l and sex-dependent traits may, in part, result from difficulties in accurately measuring Dr-l.

Experimenter-measured digit (2D or $4 \mathrm{D})$ lengths show high repeatability, and error in measurement is very small in comparison to differences in digit length between individuals (Manning et al., 1998). For values of $2 \mathrm{D}: 4 \mathrm{D}$, the error is composed of that from $2 \mathrm{D}$ and $4 \mathrm{D}$, and for Dr-l the error includes that from right 2D, right 4D, left 2D and left 4D. Simply put, it is difficult to accurately measure Dr-l. The problem is compounded when digit lengths are participant-measured rather than experimenter-measured. It is suggested that multiple measures of Dr-l be taken to reduce measurement error. For example, Hill et al. (2012) considered the relationships between 2D:4D (right 2D:4D, left 2D:4D and Dr-l) and maximal oxygen uptake in a sample of teenage boys. Digit lengths were measured from photocopies taken three times over five months. The mean Dr-1 (SD) of the three measurements was 0.014 (.024), i.e. the $S D$ was considerably smaller than that from self-measurement of digit length in the present study $(S D$, males $=.040$ to females $=.043)$. The high $S D$ values for the latter have probably arisen from elevated rates of random measurement errors. This is likely to attenuate the effect sizes reported from studies in which digit length is self-measured. Effect sizes may also be reduced by difficulties in recall of pubertal timing. Furthermore, the age when shaving commenced could be influenced by cultural expectations regarding the acceptability of facial shaving.

In conclusion, the current study found Dr-l to be sexually dimorphic (males $<$ females) with a small effect size (male-female $d=-.065$ ) which is likely to be attenuated by random measurement errors in the large self-measured sample. The sex difference was widespread across a sample of 41 nations, it was present within ethnic groups and it remained significant after the influence of age was removed. Concerning ethnicity, Dr-l differed across groups such that White and MiddleEastern peoples had low Dr-l, intermediate values were found in Asian and Chinese participants and high values among Black people. Dr-l was positively associated with the self-perceived physical developmental rate during puberty in females and males and negatively with age at menarche (females) and first shave (males) in the total sample (including seven ethnicities) and the largest ethnic group (i.e. Whites). Collectively, these findings suggest that Dr-l, in common with 2D:4D (in particular right 2D:4D), is a negative correlate of prenatal $\mathrm{T}$ and a positive correlate of prenatal E. The organizational changes associated with prenatal sex steroids are thought to relate to 
pubertal sex hormones that determine patterns of puberty. Consistent with this the authors have found evidence that Dr-l relates positively to the self-perceived speed of pubertal development. Further work is necessary to establish whether, in common with right 2D:4D, Dr-l is established prenatally and is stable during development from birth to 12 years.

Disclosure Statements.

Funding. This research received no specific grant from any funding agency, commercial entity, or not-for-profit organization.

Conflict of interest. The authors have no conflicts of interest to declare.

Ethical approval. The authors assert that all procedures contributing to this work comply with the ethical standards of the relevant national and institutional committees on human experimentation and with the Helsinki Declaration of 1975, as revised in 2008.

\section{References}

Baxter A, Wood EK, Witczak LR, Bales K and Higley JD (2020) Sexual dimorphism in Titi monkeys' digit (2D:4D) ratio is associated with maternal urinary sex hormones during pregnancy. Developmental Psychobiology 62(7), 979-991.

Bennett M, Manning JT, Cook CJ and Kilduff LP (2010) Digit ratio (2D:4D) and performance in elite rugby players. Journal of Sports Science 28(13), 1415-1421.

Breedlove SM (2010) Minireview: Organizational hypothesis: instances of the fingerpost. Endocrinology 151(9), 4116-4122.

Cohen-Bendahan CCC, van de Beek C and Berenbaum SA (2005) Prenatal sex hormone effects on child and adult sex-typed behavior: methods and findings, Neuroscience \& Biobehavioral Reviews 29(2), 353-384.

Crewther B, Cook C, Kilduff L and Manning JT (2015) Digit ratio (2D:4D) and salivary testosterone, oestradiol and cortisol levels under challenge: Evidence for prenatal effects on adult endocrine responses. Early Human Development 91(8) 451-456.

Crewther BT and Cook CJ (2019) The digit ratio (2D:4D) relationship with testosterone is moderated by physical training: Evidence of prenatal organizational influences on activational patterns of adult testosterone in physically-active women. Early Human Development 131, 51-55.

Dela Cruz C and Pereira OCM (2012) Prenatal testosterone supplementation alters puberty onset, aggressive behavior, and partner preference in adult male rats. Journal of Physiological Sciences 62(2), 123-131.

Hill R, Simpson B, Millet G, Manning J and Kilduff L (2012) Right-left digit ratio (2D:4D) and maximal oxygen uptake. Journal of Sports Sciences 30(2) 129-134.

Hönekopp J and Watson S (2010) Meta-analysis of digit ratio 2D:4D shows greater sex difference in the right hand. American Journal of Human Biology 22(5), 619-630.

Kimura D (1994) Body asymmetry and intellectual pattern. Personality Individual Differences 17(1), 53-60.

Kilduff L, Cook CJ, Bennett M, Crewther B, Bracken RM and Manning J (2013) Right-left digit ratio (2D:4D) predicts free testosterone levels associated with a physical challenge. Journal of Sports Sciences 31(6), 677-683.

Manning JT (2002) Digit Ratio: a Pointer to Fertility, Behavior and Health. New Brunswick, New Jersey, London: Rutgers University Press.

Manning JT (2011) Resolving the role of prenatal sex steroids in the development of digit ratio. Proceedings of the National Academy of Sciences USA 108(39), 16143-16144.

Manning JT, Scutt D, Wilson J and Lewis-Jones DI (1998) The ratio of 2nd to 4th digit length: a predictor of sperm numbers and concentrations of testosterone, luteinizing hormone and oestrogen. Human Reproduction 13(11), 3000-3004.

Manning JT, Churchill AJ and Peters M (2007) The effects of sex, ethnicity, and sexual orientation on self-measured digit ratio (2D:4D). Archives of Sexual Behavior 36(2), 223-233.

Manning JT and Fink B (2008) Digit ratio (2D:4D), dominance, reproductive success, asymmetry, and sociosexuality in the BBC Internet Study. American Journal of Human Biology 20(4), 451-61.

Manning JT, Trivers RL, Thornhill R and Singh D (2000) The 2nd:4th digit ratio and asymmetry of hand performance in Jamaican children. Laterality 5(2), 121-132.

Manning JT and Fink B (2011) Is low digit ratio linked with late menarche? Evidence from the BBC internet study. American Journal of Human Biology 23(4), 527-533.

Manning JT and Peters M (2009) Digit ratio (2D:4D) and hand preference for writing in the BBC Internet Study. Laterality 14(5), 528-540.

Manning J, Kilduff L, Cook C, Crewther B and Fink B (2014) Digit ratio (2D:4D): a biomarker for prenatal sex steroids and adult sex steroids in challenge situations. Frontiers in Endocrinology 5, 9.

Matchock RL (2008) Low digit ratio (2D:4D) is associated with delayed menarche. American Journal of Human Biology 20(4), 487-489. 
Mittwoch U and Mahadevaiah S (1980) Additional growth: a link between mammalian testes, avian ovaries, gonadal asymmetry in hermaphrodites and the expression of H-Y antigen. Growth 44(4), 287-300.

Muller DC, Baglietto L, Manning JT, McLean C, Hopper JL, English DR et al. (2012). Second to fourth digit ratio (2D: 4D), breast cancer risk factors, and breast cancer risk: a prospective cohort study. British Journal of Cancer 107(9), 1631-1636.

Putz DA, Gaulin SJC, Sporter RJ and McBurney DH (2004) Sex hormones and finger length - What does 2D:4D indicate? Evolution and Human Behavior 25(3), 182-199.

Ramadhani MK, Elias SG, van Noord PAH, Grobbee DE, Peeters PHM, Uiterwaal CSPM et al. (2005) Innate left handedness and risk of breast cancer: case-cohort study. BMJ 331(7521), 882-883.

Reimers S (2007) The BBC internet study: general methodology. Archives of Sexual Behavior 36(2) 147-161.

Ribeiro E Jr, Neave N, Morais RN, Kilduff L, Taylor SR, Butovskaya M et al. (2016) Digit ratio (2D:4D), testosterone, cortisol, aggression, personality and hand-grip strength: Evidence for prenatal effects on strength. Early Human Development 100, 21-25.

Schultheiss OC, Frisch M, Özbe D, Ossmann A, Schultheiss M, Lentz S et al. (2019) Implicit motives show sex-dimorphic associations with digit ratio. Motivation Science 5(4), 326-342.

Tanner JM (1990) Foetus into man: Physical growth from conception to maturity. Cambridge, MA: Harvard University Press.

Zhanbing M, Jie D, Chunyue B, Hong L, Liang P and Zhenghao H (2019) Association of CYP19A1 single-nucleotide polymorphism with digit ratio (2D:4D) in a sample of men and women from Ningxia (China). Early Human Development 132, $58-65$.

Zhang J, Yang M, Luan P, Jia W, Liu Q, Ma Z, Dang J, Lu H, Ma Q, Wang Y, Mu C and Huo Z. (2021) Associations between cytochrome P450 (CYP) gene single-nucleotide polymorphisms and second-to-fourth digit ratio in Chinese university students. Medical Science Monitor 27, e930591.

Zheng Z and Cohn MJ (2011) Developmental basis of sexually dimorphic digit ratios. Proceedings of the National Academy of Sciences USA 108(39), 16289-16294.

Cite this article: Manning JT, Fink B, Mason L, Kasielska-Trojan A, and Trivers R (2023). The effects of sex, nation, ethnicity, age and self-reported pubertal development on participant-measured right-left 2D:4D (Dr-l) in the BBC internet study. Journal of Biosocial Science 55, 383-395. https://doi.org/10.1017/S0021932022000049 